\title{
Smart Motion Detection
}

\author{
Alaeldden Abduelhadi ${ }^{1}$, Mohmmed Elnour ${ }^{2}$ \\ ${ }^{I}$ Computer and Electronic Systems Engineering, Faculty of Engineering/ University of Science and Technology, \\ Sudan \\ ${ }^{2}$ Electronics Engineering, College of Engineering/ Sudan University of Science and Technology, Sudan
}

\begin{abstract}
The development in embedded system has proved to a reliable solution in motion detection system, so motion detection is one of the key techniques in surveillance. This paper mainly focuses to improve motion detection based on low computing system "Raspberry Pi" where USB camera used to capture image when detected the unauthorized Activity using pyroelectric infrared (PIR) sensors. This system is suitable for small personal area surveillance offices/homes bank locker room, parking entrance. Whenever the motion is detected through PIR sensor the image is captured through camera and stored in the raspberry pi module and then send to email sever. The internet of things (IOT) based application used to get notifications and view the images of the motion occurrence over internet through email server, thus the system provides an innovative approach to theft detection using IOT.
\end{abstract}

Keywords: Motion detection, Raspberry Pi, USB camera, PIR Sensor, Email server, Internet of Things (IOT).

\section{Introduction}

The modern world crime has become ultra-modern too, a lot of incident occurs like robbery, stealing unwanted entrance happens. So, the security does matters in this daily life. People always remain busy in their day to day work also wants to ensure their safety of their beloved things. Sometimes they forget to look after their necessary things like keys, wallet, credit cards etc. Without these, they are unable to access their home or any place they want. To prevent these using smart motion detection system using raspberry pi. This system is not cost effective as the GSM/GPRS charges a fixed amount from each sms/call or data transfer [1]. Also, this system is not complex algorithms as the face recognition means more complex algorithms and sometimes the system may be unable to detect the visitor's identification correctly and keeps a computer chip busy for longer time [2]. If any motion is detected, the system goes into alert mode. System now sounds alarm as well as captures images of the motion happening, thus the system is an efficient camera based security system. These images are saved for later viewing reference. The user can see the thing which was detected through the internet by mobile, pc, tablets etc. As a result, the security is confirmed [3].

A street crime is the issue with which almost every government has to deal. The Closed-Circuit Television (CCTV) have been used for monitoring, recording and getting popular in whole world. CCTV systems will aid detection through its surveillance capability. Surveillance by using CCTV systems sending data like images through internet to servers but coupling these two surveillance and data transmission processes is a very difficult work. using CCTV systems data has a security problem or very challenge to handle. That cannot be used at high scale where security issues are more or difficult to handle. Also, the limitation of CCTV cameras while real time systems provide an immediate response for crime detection and the prevention [4].

Internet of things (IOT) is a concept that is a going development of the internet by which everyday 'things' objects have communication capabilities which allow them to send and receive data through connections wireless or wired and unique addressing schemes to create new applications/services and reach common goal. In particular, IOT is a new paradigm in computer science that aims at exploiting the information about the environment state in order to personalize it, that is to adapt the environment to the user preferences. The goal of the IOT is to enable everything to be connected at anytime, anyplace, with anything ideally using network. IOT technology can be created a new idea and wide development space for smart houses to provide intelligence and to improve the quality of life [5]. IOT based application can be used remotely to view the activity and get notification when motion is detected. Several applications will be presented, which are important to envisage some of the potential IOT. Some of these applications are: smart cities, smart energy, smart grid, agriculture and breeding, and pharmaceutical industry [6]. To integrate IOT in security systems to detect motion, for example every day when we are at work you will be able to monitor and get notifications if any activity happens at your home. There is no need to have machines at both ends to get the desired output thus, for home users this project will prove to be of great use as it has low energy consumption and also comes at a low cost. Also, enhancing the capabilities of these technologies and integrating them, by introducing the 'Motion Detection' system and to contribute to the current security system [7][8]. 


\section{Motivation}

The motivation is in recent developments a function smart motion detection can be done without human intervention. And the system would be an alternative for expensive security systems being used in the present day. The raspberry pi has proved to be ideal as the core of such a system. This system has to enhance the capabilities of technologies, integrating them, to introduce the 'Motion Detection' system and contribute to the current security system. Besides, the system fully automatic by increase the efficiency of the system drastically.

\section{Methodology}

The goal of this paper is to make a smart surveillance system which can be monitored by user remotely through android application. As it is connected with the system with IOT. The overall achievement from this system is a raspberry pi was used as the core of the system it receives inputs whenever the motion is detected through PIR sensor. The image is captured through camera and stored in the raspberry pi module, which producing the output to monitor a security system from a location away from the surveillance area through android device. In addition, used Raspbian wheezy operating system as our operating system with the suitable software python programming language.

\section{System Materials}

The block of smart motion system shown in Fig. 1, in this system when the PIR sensor senses motion it gives a signal to the raspberry pi to take the image through USB camera and then send the image to email sever via the internet, so that the user can see the image that was taken. The system materials consist two parts: The hardware material part, and the software materials part.

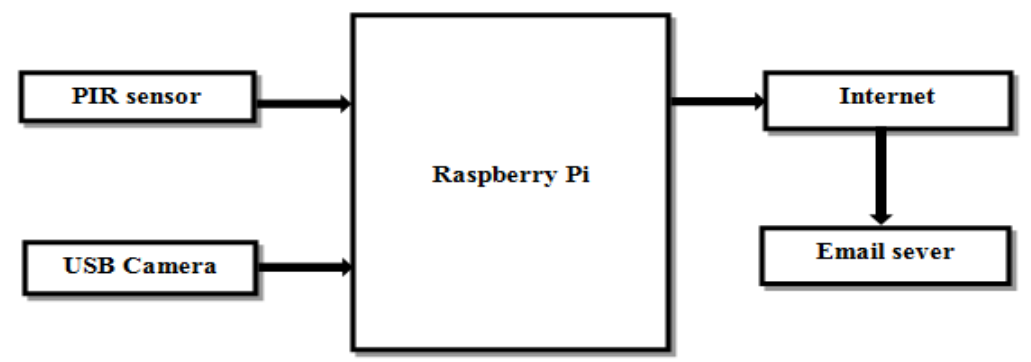

Fig. 1. Block diagram of smart motion system

\subsection{Hardware materials}

\subsubsection{PIR Sensor}

This section explains the main components used in motion system.

PIR sensors is a pyroelectric device that sense motion by measuring changes in the infrared heat levels. PIR sensor is small, cheaper, low power consumption, easy to use. For that reason, it's commonly found in appliances and gadgets used in homes or businesses. When motion is detected used the breakout board to convert the signal into a longer digital signal, and output is a high signal on its output pin. This logic signal can be read by any embedded processor or used to drive a transistor to switch a higher current load [9].

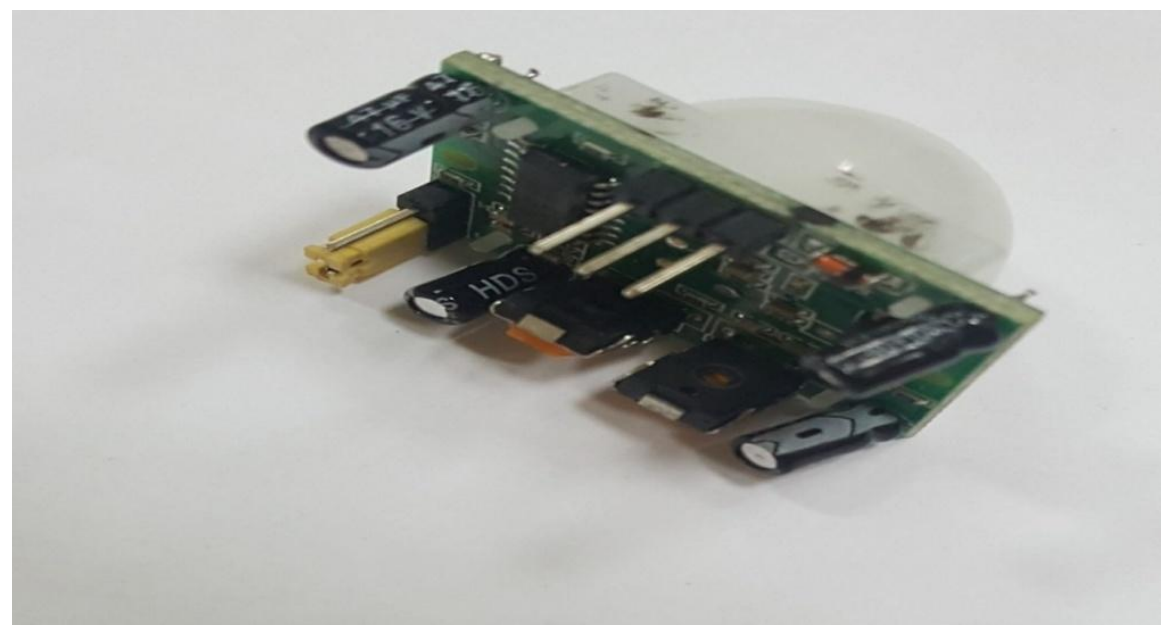

Fig. 2. PIR sensor 
Fig. 2 shows the PIR sensor it has three pins (VCC, GND and Output pin), it's generates $+5 \mathrm{v}$ and $-5 \mathrm{v}$ sine signal when any human or animal passes in front of the sensor or any movement is detected, the output of PIR sensor can be adjusted. For max, it can create an output signal for approximately 1.2 seconds [10]. This is more than enough to detect the signal.

\subsubsection{USB Camera}

USB Cameras is a camera that feeds or streams its image in real time to or through a computer to a computer network. It's imaging cameras that use USB technology (USB 2.0 or 3.0) to transfer image data. It designed to easily interface with computer systems by using the same USB technology that is found on most computers. Fig. 3 shows USB camera that low manufacturing cost and their high flexibility, making them the lowest-cost. Used a standard USB webcam to take pictures and video on the raspberry pi connected by a USB cable, or similar cable, when "captured" by the raspberry pi, the pictures and video are saved or sent to other networks via systems such as the internet, and emailed as an attachment.

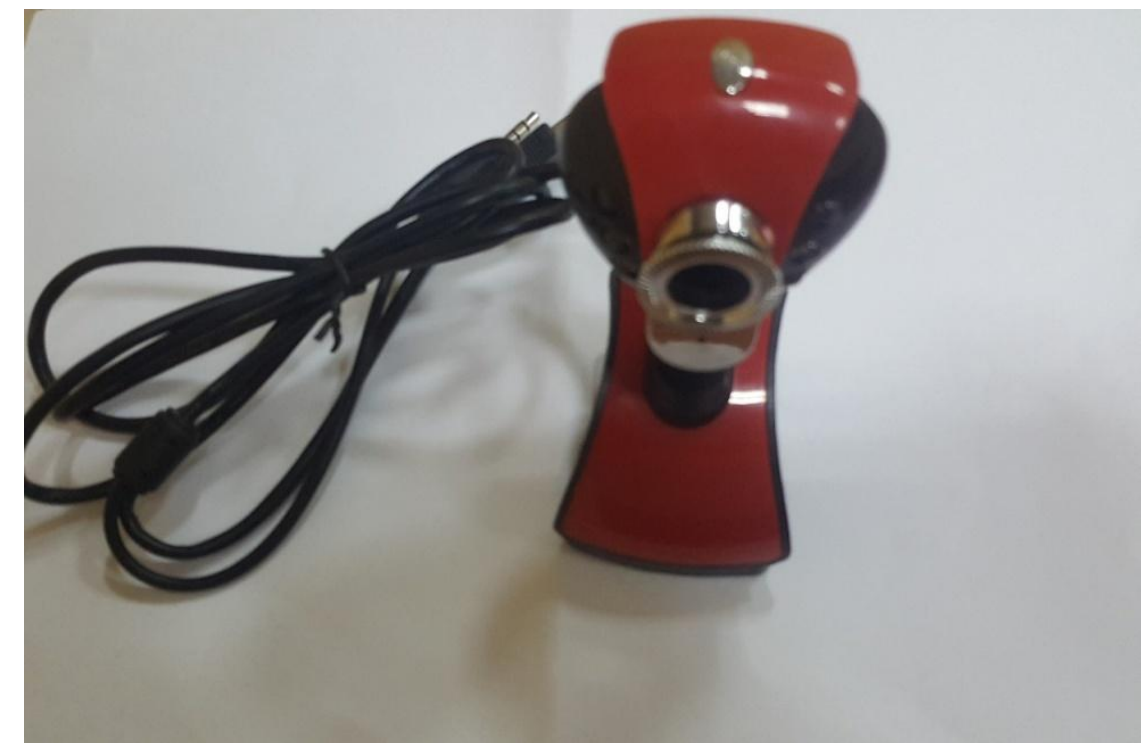

Fig. 3. USB camera

\subsubsection{Raspberry Pi 3 Model B}

The raspberry pi is a single board, low cost, credit card sized and small computer to capable of perform different functionalities such as identification and access control, electronics payment system, health application etc. Raspberry pi is a low power computer system running Linux (or several other operating systems) on an ARM processor architecture. The system boots from an SD card and an 8 GB SD card is more than enough to store the operating system and lots of data. It does not include a built-in hard disk or solid-state drive, but uses an SD card for booting and persistent storage. The power requirements are very modest and a simple $5 \mathrm{~V}$ phone charger with micro USB plug is enough to run the system. The software is very stable (many units have been running non-stop for several months) and there are many general-purpose I/O pins (GPIO) available for interfacing to external devices. The device is a reasonably powerfull computer on a credit card sized PCB [11]. The raspberry pi 3 Model B is the $3^{\text {rd }}$ generation raspberry pi. It's more powerful processer, 10x faster than the $1^{\text {st }}$ raspberry pi. Also, it adds wireless LAN \& Bluetooth connectivity making it the ideal solution for powerful connected designs. Fig. 4 shows raspberry pi 3 it has a Broadcom BCM2835 system on a Chip, this chip is a 32 bit which includes an ARM1176JZF-S Processor. Typical clock size is $700 \mathrm{MH}$, preforming at approximately 40 MFLOPS Can be overclocked to $1 \mathrm{GHz}$ without any issues, Includes Video Core IV graphics processor w/ 1 billion pixels per second, Model A has $256 \mathrm{MB}$ and Model B has 512MB of RAM is built into the board, not replaceable or upgradeable also Include multiple built on I/O ports (100 MB/s Ethernet port HDMI port and RCA port Audio Jack) [12] [13]. The big advantage of the raspberry pi was that it could be put it in places we couldn't put a PC. The raspberry pi being small as a credit card server still has the capabilities of working as a normal computer it can play 10S0p resolution videos without lagging. 


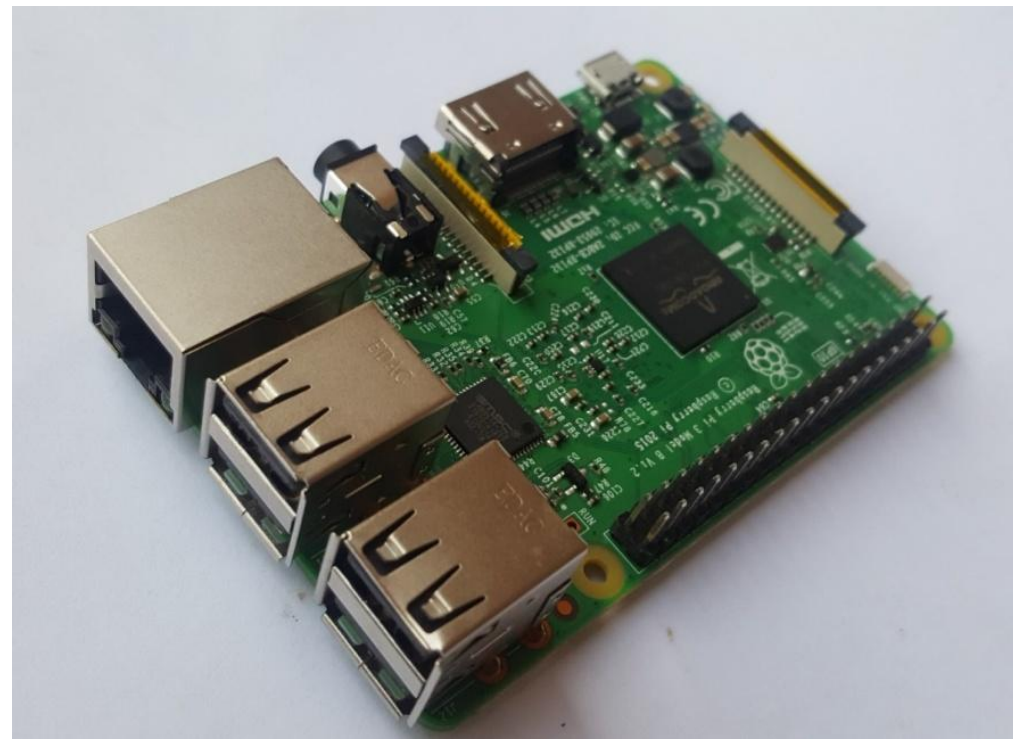

Fig. 4. Raspberry pi 3 model B

\subsection{Software Materials}

There are many software materials like compilers and programs that have been used during the development of the system. An operating system is a program that makes it easier for the end user to use the underlying hardware. Raspberry pi supports multiple Linux distributions. Because Linux code is publicly available, different organizations have made slight changes to it and distributed it. This has led to different distributions (versions), including Red Hat, Fedora, Debian, Arch, Ubuntu and open SUSE. Some companies sell their distributions and provide paid-for support, whereas others are completely free [14]. Free Software Foundation uses the name GNU/Linux, which has led to some controversy. Raspbian is based on the Debian distribution with some customizations for the raspberry pi. More programming languages can be installed with proper support tools. The installed ARMv6 also handle many languages: (Python, C, C++, Java, Ruby and Scratch). Python is high level programming language, widely used general-purpose. It's supports multiple programming paradigms, including object oriented program, imperative and functional programming or procedural styles and provides constructs intended to enable clear programs on both a large and small scale. Its syntax allows programmers to express ideas in minimal lines of code than would be possible in the high-level languages such as Java or C++.Python interpreter runs in an interactive shell to execute individual commands, or as a command line program to execute standalone scripts [15].

\section{System Implementation}

Hardware connect the USB camera and PIR sensor to the raspberry pi shown in Fig. 5. Use three female-to-female jumper cables, to connect each of the PIR sensor's connectors to the appropriate pins on the raspberry pi. Then connect the top one labelled VCC on the PIR sensor to the $5 \mathrm{~V}$ pin on the raspberry pi GPIO [pin 2], connect the middle one labelled OUT to GPIO pin 4, and connect the bottom one labelled GND to a ground pin also marked GPIO [pin 6].

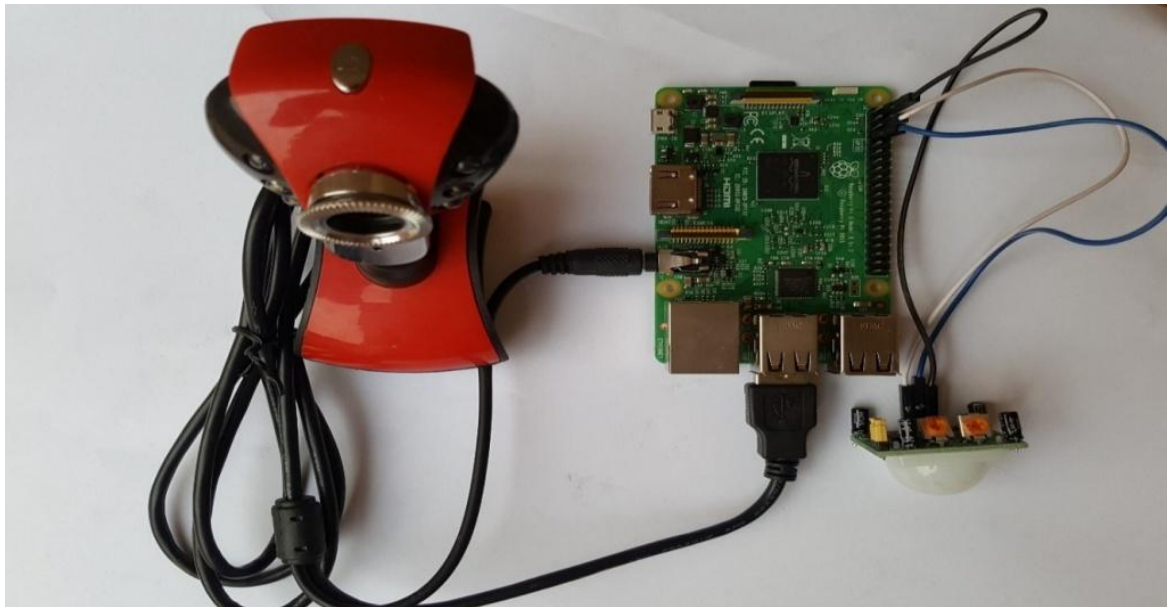

Fig. 5. Hardware implementation 
The software implementation process followed these steps: used hardware peripherals like (Screen, Keyboard and mouse) to communicate the raspberry pi. It was done by connecting the raspberry pi to the internet using the Ethernet interface or Wifi-Dongle. The reason behind running the raspberry pi headless is the power that is consumed by the raspberry pi peripherals. Fig. 6 shows the flow chart of designed system. At first the system detects motion and automatically raspberry pi take image and save it into raspberry pi memory. After that image is sent to the administrator email. At last when the email is opened the captured image will be found.

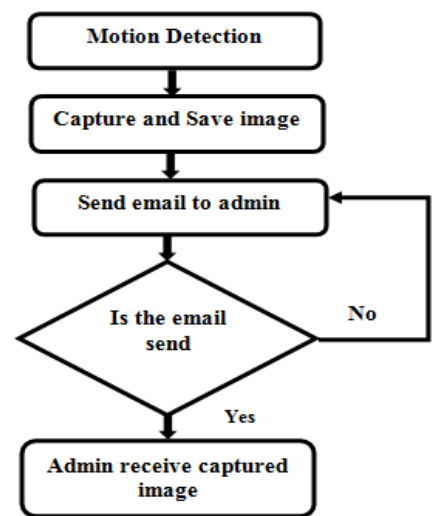

Fig.6. Flow chart of the designed System

\section{Results}

The results of the system were started by coding for the main units of the system using python programming language. Many modifications and enhancements have been made to the codes and the circuit for the system were ready and the final installation setup shown in Fig. 7. When runs the python code whenever there is a movement it detected by the PIR sensor, an image is captured and saved into the raspberry pi module as shown in Fig. 8. then send the image to email server and will get an image. Fig. 9 shows the admin email view the captured image.
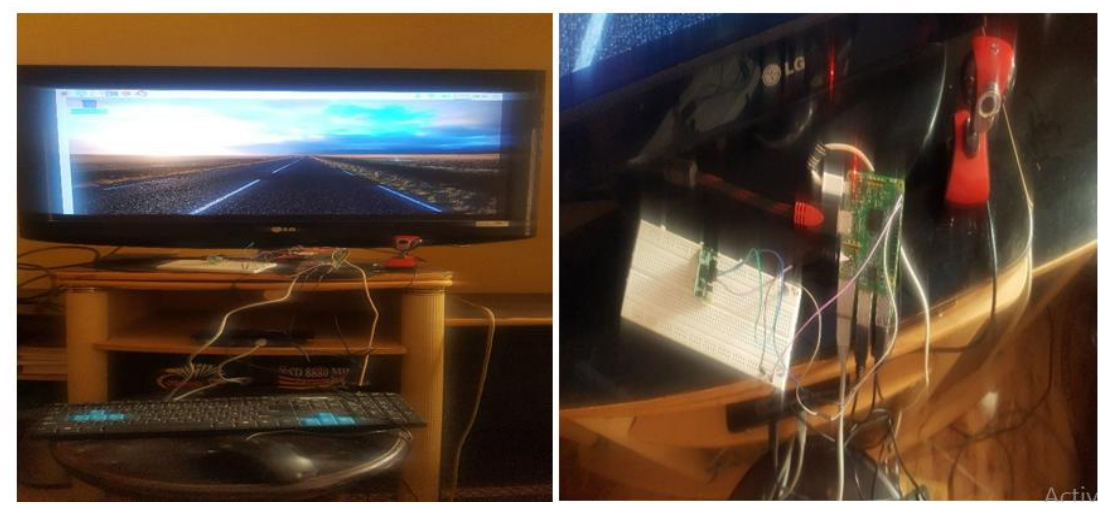

Fig. 7. Raspberry pi final installation setup

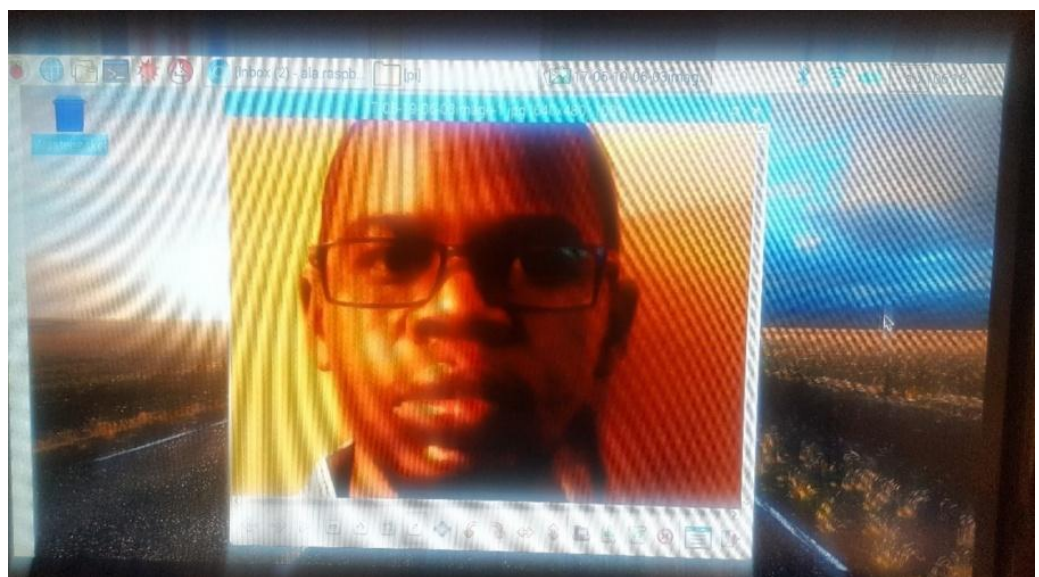

Fig. 8. Image that saved into raspberry pi from USB camera 


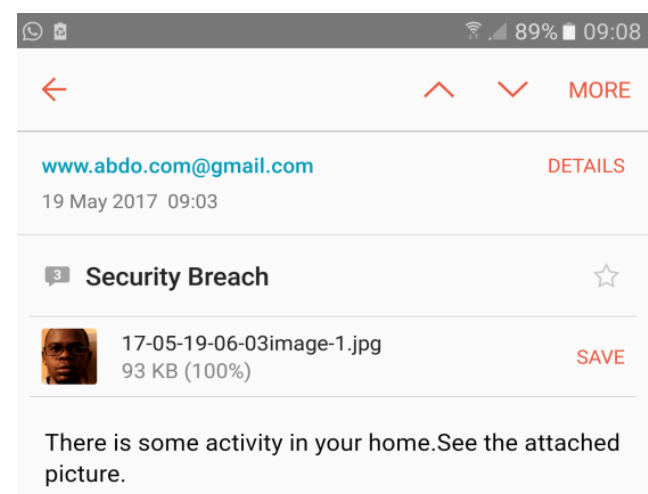

Fig. 9. Admin email received captured image

\section{Conclusion}

The main objective of the project, which is the design and implementation of smart motion detection system to be displayed through web based application has been done successfully. The system can be used in different environments and several places like houses, banks, hospitals, labs and other automated systems, which dramatically reduce the hazard of unauthorized entry. But the system needs to be monitor always that the internet bills are paid in due time to keep connected to provide information to the user about what is happening in surveillance area by sending notification to email server. This system would be an alternative for expensive security systems being used.

\section{Acknowledgement}

This paper would not have been possible without the inspiration and support of a number of wonderful individuals, my thanks and appreciation to all of them for being part of this journey and making this thesis possible. My thanks are due to Karary University and to the College of Engineering. Spatially Electrical Engineering Department. My sincere gratitude to Dr. Salah who supervised this paper whose advice was very helpful and important. And has made every effort in order to reach this stage. My thanks also to the staff working in Sudan University of Science and Technology. Thanks again to all who helped me.

\section{References}

[1]. Zhao, Yanbo, and Zhaohui Ye. "A low cost GSM/GPRS based wireless home security system." IEEE Transactions on Consumer Electronics 54.2 (2008).

[2]. Chowdhury, MdNasimuzzaman, MdShibleeNooman, and SrijonSarker. "Access Control of Door and Home Security by Raspberry Pi Through Internet." Int. J. Sci. Eng. Res 4 (2013): 550-558.

[3]. Patel, Urvija J., and Nehal G. Chitaliya. "Smart Sensing System using Internet of Things."

[4]. Rayte, Swapnali, et al. "Crime Monitoring and Controlling System by Mobile Device."

[5]. Piyare, Rajeev. "Internet of things: ubiquitous home control and monitoring system using android based smart phone." International Journal of Internet of Things 2.1 (2013): 5-11.

[6]. Prasad, Sanjana, et al. "Smart Surveillance Monitoring System Using Raspberry PI and PIR Sensor." Int. J. Comput. Sci. Inf. Technol 5 (2014): 7107-7109.

[7]. Ansari, Aamir Nizam, et al. "An Internet of things approach for motion detection using Raspberry Pi." Intelligent Computing and Internet of Things (ICIT), 2014 International Conference on. IEEE, 2015.

[8]. Friess, Peter. Internet of things: converging technologies for smart environments and integrated ecosystems. River Publishers, 2013.

[9]. Yun, Jaeseok, and Sang-Shin Lee. "Human movement detection and identification using pyroelectric infrared sensors." Sensors 14.5 (2014): 8057-8081.

[10]. Hossain, Md Khaled, and Sayed SamialHaq. "Detection of Car Pre-Crash with Human, Avoidance System \& Localizing through GSM." International Journal of Scientific and Research Publications 3.7 (2013): 1-4.

[11]. Holton, Jon, and Tim Fratangelo. "Raspberry Pi Architecture." Raspberry Pi Foundation, London, UK (2012).

[12]. Richardson, Matt, and Shawn Wallace. Getting started with raspberry PI. " O'Reilly Media, Inc.", 2012.

[13]. Vaidya, Rohit Ratnakar. "Efficient Embedded Surveillance System with Auto Image Capturing and Email Sending Facility." International Journal of Technical Research and Applications (2015): 109-112.

[14]. Robinson, Andrew, and Mike Cook. Raspberry Pi Projects. John Wiley \& Sons, 2013.

[15]. Jadhav, Gaurav, Kunal Jadhav, and Kavita Nadlamani. "Environment Monitoring System using Raspberry-Pi." (2016). 